\title{
Comparación de la dureza superficial de resinas de nanotecnología, según el momento del pulido: in vitro.
}

\author{
Comparison of surface hardness of nanotechnology composites according to polishing time: in vitro
}

Rolan Suarez ${ }^{l, a}$, Felipe Lozano ${ }^{2, b, c}$.

RESUMEN

Objetivo: Se comparó el efecto del pulido inmediato y después de 24 horas de la dureza superficial de resinas de nanotecnología. Material y métodos: Confeccionamos 32 cilindros según la norma ISO 4049 y colocamos resinas divididos en 2 grupos de 16 cada uno. Un grupo de resina nanohíbrida y otro de nanorrelleno. La mitad de cada grupo fue pulida al momento y la otra mitad a 24 horas de su polimerización. La microdureza Vickers se determinó con una carga de $500 \mathrm{~g}$ durante $10 \mathrm{~s}$. Los datos fueron analizados con la prueba T-Student. Resultados: El pulido después de $24 \mathrm{~h}$ obtuvo superficies con mayor dureza superficial respecto al pulido inmediato siendo estadísticamente significativo $(\mathrm{P}=0,0001)$. La resina nanohíbrida presentó mayor dureza superficial que nanorrelleno para el pulido inmediato y el pulido a las 24 horas siendo en ambos casos estadísticamente significativo. Conclusión: El pulido después de 24 horas de su polimerización presento valores con mayor dureza superficial para la resina nanohíbrida y de nanorelleno.

\section{Palabras clave: DUREZA, PULIDO DENTAL.}

\section{SUMMARY}

Objective: To evaluate the polishing time effects over superficial microhardness in nanotechnology composites. Methods: Two kinds of composites were used: nanohybrid and nanofilled. 32 standardized samples were prepared which were distributed in two groups each one $(n=16)$ according to polishing time. Both groups were subdivided in two, according to kind of composite, nanohybrid and nanofilled ( $n=8)$, the first group was polished immediately while the second one polished 24 hours after light cured. Vickers microhardness was determined through 5 indentations with a $500 \mathrm{~g}$ of load and $10 \mathrm{~s}$ of dwell time. Statistical analysis was made by Student T-Test. Results: Polishing after 24 hours obtained significantly higher superficial hardness $(P=0.0001)$. Nanohybrid composite showed higher hardness relating to nanofilled composite. Conclusion: Polishing after 24 hours produced surfaces with higher superficial hardness.

Keywords: HARDNESS, DENTAL POLISHING.

\footnotetext{
Facultad de Odontología, Universidad Nacional Federico Villarreal. Lima, Perú.

2 Facultad de Odontología, Universidad Nacional Mayor de San Marcos. Lima, Perú.

a Cirujano Dentista.

b Maestría en Estomatología.

c Profesor del Departamento de Estomatología Rehabilitadora.
} 


\section{INTRODUCCIÓN}

Los materiales de restauración estéticos han mejorado desde su introducción a la odontología en la década de los 60 y con el pasar de los años las resinas compuestas han ido alcanzando un rol protagónico e importante frente a las amalgamas (1). Pese a los avances logrados las resinas compuestas presentan aún deficiencias que limitan sus aplicaciones clínicas, siendo principalmente la respuesta frente a los fenómenos de desgaste y su contracción de polimerización los que nos conllevan a fracasos clínicos durante su uso (2). Las investigaciones orientadas a mejorar la resistencia al desgaste y disminuir la contracción de polimerización de las resinas compuestas se han centrado principalmente en variaciones del tamaño, composición y distribución del relleno dentro de la matriz de resina, llegando en los últimos años a una nueva tecnología de relleno de tamaño nanométrico que al poseer un menor tamaño de partícula evidenciaron un menor grado de contracción durante la polimerización y brindaron al material no solo una mejor dureza sino también una mejor calidad de superficie y mayor capacidad de pulido (2-4). La dureza superficial del material tiene gran importancia en el éxito clínico de la restauración, ya que mientras mayor sea, brindará al material una mejor resistencia al desgaste y al rayado $(4,5)$. Para lograr mejorar esta propiedad mecánica las superficies de los materiales compuestos son sometidos a procedimientos de pulido debido a que superficies rugosas disminuyen su resistencia además de facilitar la adhesión bacteriana, lo que puede resultar en inflamación gingival, tinción superficial e incluso caries secundaria $(6,7)$.

Por tanto un acabado y pulido adecuado es necesario para establecer una textura suave, brillante y con contornos anatómicos óptimos que garanticen el éxito y longevidad de la restauración (8).

El momento del pulido podría tener un efecto en las propiedades físicas del material de restauración sobre todo en su resistencia al desgaste o dureza, ya que dependerá mucho del grado de polimerización que presente el material y las distribución que tengan la partículas de carga inorgánica dentro de la matriz resinosa, es por eso que el mal manejo de este procedimiento podría llevarnos incluso propensamente al fracaso clínico $(9,10)$.
Algunos investigadores y fabricantes proponen una espera de 24 horas y hasta de una semana para realizarlo, sin embargo la mayoría de profesionales lo realiza inmediatamente por diferentes motivos como la necesidad inmediata de mayor estética por parte del paciente, el menor tiempo clínico para el operador, el no retorno del paciente a la consulta, entre otros (11).

El objetivo del presente trabajo fue determinar el momento adecuado del pulido de una restauración con resinas de nanotecnología y el efecto de este momento sobre su dureza superficial.

\section{MATERIAL Y MÉTODOS}

Se emplearon para este estudio dos tipos de resina: una resina nanohíbrida (3M ESPE Filtek Z250 XT) y una resina de nanorrelleno (3M ESPE Filtek Z350 $\mathrm{XT}$ ). Ambos materiales restauradores fueron de color A2.

Se elaboraron 32 muestras en cilindros estandarizados (6 mm altura x $6 \mathrm{~mm}$ de diámetro), siguiendo las especificaciones de la norma ISO 4049, para la obtención de las muestras se utilizó un molde que cumplía los requisitos de la norma de estandarización, siguiendo las recomendaciones del fabricante las resinas fueron introducidas dentro del molde en tres incrementos de $2 \mathrm{~mm}$ cada uno, después de introducir el último incremento se colocó sobre el molde una tira celuloide y sobre ella una lámina portaobjeto para garantizar que las superficies tanto superior como inferior queden paralelas entre sí, posteriormente las muestras se polimerizaron desde la parte superior del molde con una lámpara LED (Gnatus) de acuerdo a las instrucciones del fabricante a una intensidad de $1200 \mathrm{mw} / \mathrm{cm} 2$ verificado con un radiómetro (radiómetro de fotocurado Dentamérica, USA) y durante un tiempo de $20 \mathrm{~s}$.

Las muestras fueron distribuidas según el tipo de resina en nanohíbridas y nanorrelleno en dos grupos de 16 cada uno. A su vez estos grupos se subdividieron en dos según el momento del pulido, la mitad fueron pulidos inmediatamente mientras que la otra mitad 24 horas después.

El pulido se realizó con discos de óxido de aluminio (Sof-lex) de grano grueso a grano fino, cada 
disco se utilizó con movimientos circulares con una ligera presión durante 20 segundos con una pieza de baja velocidad (Kavo, Alemania), este procedimiento se realizó por un operador al que se denominó "ciego" quien garantizó un procedimiento aleatorio y que no pueda sesgar los resultados.

Todos los grupos se almacenaron en agua destilada a una temperatura de $37^{\circ} \mathrm{C}$ por una semana.

Por último se realizó la medición de la dureza superficial de los grupos mediante el ensayo Vickers, usando un microdurómetro (ZHV Zwick Roell). La carga aplicada fue de $500 \mathrm{~g}$ con un tiempo de permanencia de $10 \mathrm{~s}$, se realizaron 5 indentaciones en cada muestra con la misma distancia entre ellas y con no más de $1 \mathrm{~mm}$ adyacentes a los márgenes de la

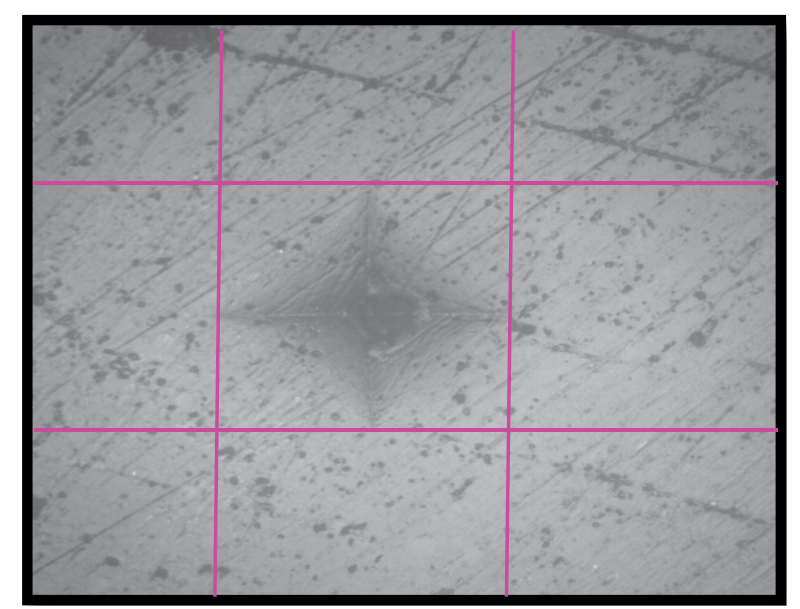

Figura 1. Indentación Resina Nanohíbrida (Carga = 500gTiempo $=10 \mathrm{~s})$

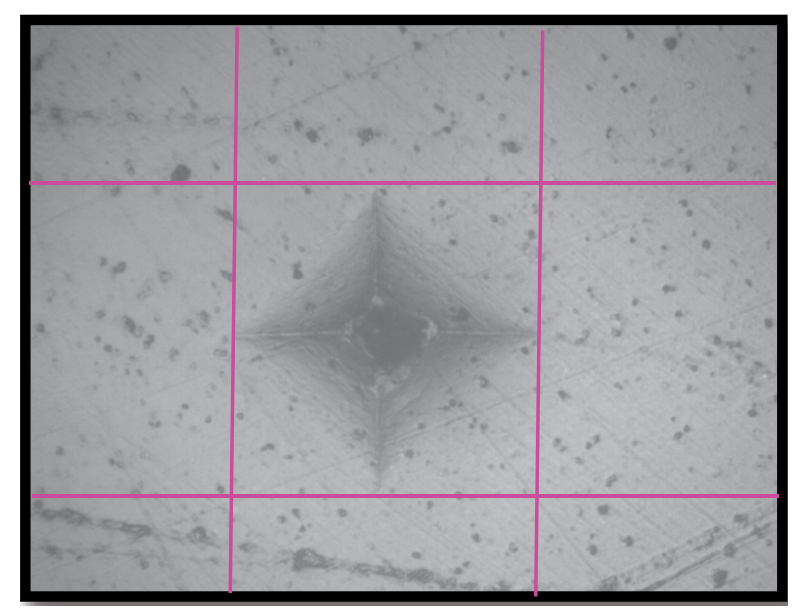

Figura2. Indentación Resina Nanorrelleno Carga $=500 \mathrm{~g}-$ Tiempo $=10 \mathrm{~s})$ muestra. La dureza se determinó mediante la división de área de la huella dejada sobre la carga aplicada, tanto para la resina nanohíbrida (Figura 1) como para la resina de nanorrelleno (Figura 2).

\section{RESULTADOS}

Se evaluaron 32 muestras, siendo 8 muestras por cada grupo. Las resinas de nanorrelleno con pulido inmediato mostraron una dureza media de $86,45 \mathrm{HV}$ (Tabla 1), mientras que cuando fueron pulidas $24 \mathrm{~h}$ después el resultado de dureza media fue de 91,20 HV (Tabla 2).

Tabla 1. Dureza superficial de la resina de nanorrelleno pulida inmediatamente.

\begin{tabular}{ccccccc}
\hline Resina & $N^{\circ}$ & Media & D.S. & Mediana & Mínimo & Máximo \\
& & & & & & \\
Nanorrelleno & 8 & 86,450 & 1,360 & 86 & 85,2 & 89,6 \\
\hline
\end{tabular}

Tabla 2. Dureza superficial de una resina de nanorrelleno pulida 24 horas después.

\begin{tabular}{ccccccc}
\hline Resina & $N^{\circ}$ & Media & D.S. & Mediana & Mínimo & Máximo \\
Nanorrelleno & 8 & 91,200 & 1,031 & 90,8 & 90,2 & 92,6 \\
\hline
\end{tabular}

Para las muestras de resina nanohíbrida, las que tuvieron pulido inmediato mostraron un valor de dureza media de 92,52 HV (Tabla 3), mientras que con un pulido después de $24 \mathrm{~h}$ el resultado fue de 100,65 HV (Tabla 4).

Tabla 3. Dureza superficial de una resina nanohíbrida pulida inmediatamente.

\begin{tabular}{ccccccc}
\hline Resina & $\mathrm{N}^{\circ}$ & Media & D.S. & Mediana & Mínimo & Máximo \\
Nanohíbrida & 8 & 92,525 & 0,575 & 92,5 & 91,8 & 93,6 \\
\hline
\end{tabular}

Tabla 4. Dureza superficial de una resina nanohíbrida pulida 24 horas después.

\begin{tabular}{ccccccc}
\hline Resina & $N^{\circ}$ & Media & D.S. & Mediana & Mínimo & Máximo \\
Nanohíbrida & 8 & 100.650 & 0.812 & 100.7 & 99 & 101.8 \\
\hline
\end{tabular}


Los resultados se sometieron a la prueba T-Student con nivel de significancia de 95\% para comparar las medias de dureza superficial. La resina de nanorrelleno mostró mayores valores de dureza superficial cuando el pulido se realizó $24 \mathrm{~h}$ después de su polimerización siendo estadísticamente significativo $(\mathrm{P}=0,0001)$ (Tabla 5). Lo mismo ocurrió para la resina nanohíbrida que mostro mayores valores de dureza superficial cuando el pulido se realizó 24 h después siendo también estadísticamente significativo $(\mathrm{p}=0,00001)$ (Tabla 6$)$.

Tabla 5. Comparación de la dureza superficial de una resina de nanorrelleno pulida inmediatamente y pulida 24 horas después.

\begin{tabular}{lcccccc}
\hline Momento de pulido & $\mathrm{N}^{\circ}$ & Media & E.S. & D.S. & $\begin{array}{r}\text { IC } \\
95 \%\end{array}$ \\
\hline \multicolumn{1}{c}{ Inmediato } & 8 & 86,5 & 0,5 & 1,4 & 85,3 & 87,6 \\
$\quad 24$ horas & 8 & 91,2 & 0,4 & 1,0 & 90,3 & 92,1 \\
Combinado & 16 & 88,83 & 0,68 & 2,72 & 87,38 & 90,27 \\
Diff & & $-4,75$ & 0,60 & & $-6,04$ & $-3,46$ \\
\hline
\end{tabular}

$\mathrm{t}=7.8738, \quad \mathrm{P}=0.0001$

Tabla 6. Comparación de la dureza superficial de una resina Nanohíbrida pulida inmediatamente y pulida 24 horas después.

\begin{tabular}{ccccccc}
\hline Momento del pulido & $\mathrm{N}^{\mathrm{o}}$ & Media & E.S. & D.S. & IC 95\% & \\
\hline Inmediato & 8 & 92,53 & 0,20 & 0,58 & 92,04 & 93,01 \\
24 horas & 8 & 100,65 & 0,29 & 0,81 & 99,97 & 101,33 \\
combinado & 16 & 96,588 & 1,063 & 4,250 & 94,323 & 98,852 \\
Diff & & $-8,125$ & 0,352 & & $-8,880$ & $-7,370$ \\
\hline
\end{tabular}

$\mathrm{t}=23,0884, \mathrm{P}=0,00001$

Los resultados evidenciaron que la resina nanohíbrida mostró mayores valores de dureza superficial respecto a la de nanorrelleno cuando el pulido se realizó inmediatamente, siendo esta diferencia estadísticamente significativa $(\mathrm{P}=0,00001)$ (Tabla 7). Los mismos resultados se obtuvieron cuando el pulido se realizó $24 \mathrm{~h}$ después, la resina nanohíbrida mostró una dureza superficial más alta respecto de la de nanorrelleno siendo también estadísticamente significativa $(\mathrm{p}=0,00001)$ (Tabla 8). El valor medio más alto de dureza superficial se obtuvo con las resina nanohíbrida y cuando el pulido se realizó $24 \mathrm{~h}$ después de la polimerización del composite, 100.65HV (Tabla 4).
Tabla 7. Comparación de la dureza superficial de una resina de nanorrelleno y una resina nanohíbrida pulidas inmediatamente.

\begin{tabular}{lcccccc}
\hline Resina & $\mathrm{N}^{\mathrm{o}}$ & Media & E.S. & D.S. & IC 95\% & \\
\hline Nanohíbrida & 8 & 92,53 & 0,20 & 0,58 & 92,04 & 93,01 \\
Nanorrelleno & 8 & 86,45 & 0,48 & 1,36 & 85,31 & 87,59 \\
combinado & 16 & 89,49 & 0,82 & 3,30 & 87,73 & 91,24 \\
Diff & & 6,07 & 0,52 & & 4,96 & 7,19 \\
\hline
\end{tabular}

$\mathrm{t}=11,6395, \quad \mathrm{P}=0,00001$

Tabla 8. Comparación de la dureza superficial de una resina de nanorrelleno y una resina nanohíbrida pulidas 24 horas después.

\begin{tabular}{lcccccc}
\hline Resina & $\mathrm{N}^{\mathrm{o}}$ & Media & E.S. & D.S. & IC 95\% & \\
\hline Nanohíbrida & 8 & 100,65 & 0,29 & 0,81 & 99,97 & 101,33 \\
Nanorrelleno & 8 & 91,20 & 0,36 & 1,03 & 90,34 & 92,06 \\
combinado & 16 & 95,93 & 1,24 & 4,96 & 93,28 & 98,57 \\
Diff & & 9,45 & 0,46 & & 8,45 & 10,45 \\
\hline
\end{tabular}

$\mathrm{t}=20,3635, \quad \mathrm{P}=0,00001$

\section{DISCUSIÓN}

Las necesidades estéticas del paciente muchas veces han conducido al clínico a realizar restauraciones con resinas compuestas aun en superficies que soportarán directamente la acción de grandes cargas masticatorias, especialmente las caras oclusales de los dientes posteriores a nivel de las cúspides de trabajo y las funcionales del sector anterior, sin embargo, el alto nivel de desgaste que estas presentan, producto de la carga que recae sobre ellas en muchos casos podría conllevar a la disminución de la dimensión vertical y afectar el equilibrio del sistema estomatognático (12).

La dureza determina el grado de deformación de un material, su resistencia a la indentación y se acepta como una propiedad importante y un parámetro valioso en la comparación con la estructura dental. Los valores de dureza de esmalte y dentina en unidades Vickers se expresan como 348HV y $80 \mathrm{HV}$ respectivamente (13-15).

Este estudio tuvo como objetivo medir el efecto del pulido en la dureza superficial de dos resinas de nanotecnología indicadas como material de restauración para dientes posteriores. En primera instancia, se determinó la dureza de las resinas 
compuestas según el momento del pulido, parámetro útil para analizar la resistencia a la indentación de la resina, que puede relacionarse con su resistencia al desgaste y su capacidad de pulido y brillado $(16,17)$.

Los resultados revelaron que pulir el material restaurador aumento su dureza superficial independientemente del momento en el que se realice. Estos resultados fueron corroborados por Park y col quienes también verifican la influencia del pulido en la dureza de una resina compuesta, encontrando además que aunque se puede obtener una superficie más lisa con tiras celuloide, la capa superficial se compone principalmente de matriz orgánica, siendo por lo tanto menos denso que la capa subyacente (18). Por otra parte el pulido puede proporcionar una superficie más resistente a la deformación y si el pulido se realiza inmediatamente esta polimerización incompleta podría convertir al compuesto en uno más susceptible a los efectos del calor, aunque el 75\% de la polimerización se produce en los primeros 10 minutos, este proceso de curado puede continuar durante un periodo de 24 a 30 horas $(19,20)$.

El presente estudio concluyo que el pulido después de las 24 horas aumentó significativamente la dureza superficial de las resinas compuestas, resultados corroborados por Chinelatti y col quienes encontraron un aumento significativo de dureza en relación al tiempo de pulido siendo superior después de las 24 horas en comparación con el pulido inmediato (20).

Otra investigación que encontraron resultados similares fueron realizados por Yazici y col quienes tuvieron como objetivo encontrar el efecto de un retraso de 24 horas en el pulido sobre la rugosidad superficial, dureza y brillo de los materiales compuestos, obteniendo como resultado que el acabado y pulido tardío posterior a las 24 horas aumentó significativamente la dureza de todos los materiales (21).

La mayor parte del material polimerizado posteriormente absorbe agua y genera una reacción acido base que sirve para endurecer y fortalecer los polímeros de matriz. Con el aumento del tiempo la fase de matriz superficial madura y se endurece, la disminución de la matriz de la matriz durante el acabado y pulido retardado dan valores de dureza superiores tal como se encontró para ambos tipos de resinas compuestas utilizadas en el presente estudio, resultados que fueron ratificados por Yap y Col quienes encontraron mayor dureza superficial de las muestras en relación al mayor tiempo de espera para su pulido después de su almacenaje en agua destilada a $37^{\circ} \mathrm{C}$ para simular el ambiente de la cavidad oral (22).

Venturini y col informaron que el pulido inmediato no produjo una influencia negativa en la rugosidad, dureza y microfiltración del material compuesto en comparación con el pulido posterior a las 24 horas, ellos atribuyen sus resultados a que el pulido tiene un efecto sobre la dureza pero es muy dependiente del sistema y la técnica de pulido además del material, ellos no encontraron diferencias significativas en sus resultados llegando incluso a recomendar el pulido inmediato, ya que reducirá el número de sesiones y se dará mayor comodidad al paciente (23).

En otro estudio Venturini y col contrariamente a nuestros hallazgos, encontraron que los especímenes con el pulido tardío mostraron menor dureza en comparación con las muestras que fueron pulidas inmediatamente, incluso después de un almacenamiento de un año los autores atribuyeron este resultado a la perdida de propiedades superficiales después de su polimerización utilizando una técnica de pulido inmediato. También recomendaron pulido inmediato ya que este procedimiento brindara comodidad al paciente y reducirá costos y tiempo del operador (24).

En conclusión según los hallazgos del estudio, el pulido de la resina nanohíbrida y de nanorrelleno después de 24 horas de su polimerización presentaron valores con mayor dureza superficial con respecto al pulido inmediato, siendo significativo estadísticamente.

\section{CORRESPONDENCIA:}

Rolan Suárez

Jr. Manco II 274, Urb. Maranga,

San Miguel, Lima, Perú.

Correo electrónico: jhosuat12@gmail.com 


\section{REFERENCIAS BIBLIOGRÁFICAS}

1. Morgan M. Finishing and polishing of direct posterior resin restorations. Pract Proced Aesthet Dent. 2004;16(3):211-7.

2. Setcots JC, Tarim B, Susuki S. Surface Finish produced on resin composites by new polishing systems. Quintessence Int. 1999;30(3):169-73.

3. Anusavice KJ, Antonson SA. Finishing and polishing materials in Philip's Science of dental materials. 11th ed. Philadephia: WB Saunders Co; 2003. p. 352-3.

4. O'Brien WJ, Johnston WM, Fanian F, Lambert S. The surface roughness and gloss of composites. J Dent Res. 1984; 63(5):685-8.

5. Da Costa J, Ferracane J, Paravina RD, Mazur RF, Roeder L. The effect of different polishing systems on surface roughness and gloss of various resin composites. J Esthet Restor Dent. 2007;19(4):214-24.

6. Paravina RD, Roeder L, Lu H, Vogel K, Powers JM. Effect of finishing and polishing procedures on surface roughness, gloss and color of resin-based composites. Am J Dent. 2004;17(4):262-6.

7. Stanford WB, Fan PL, Wozniak WT, Stanford JW. Effect of finishing on color and gloss of composites with different fillers. J Am Dent Assoc. 1985;110(2):211-3.

8. Turkun LS, Turkun M. The effect of one-step polishing system on the surface roughness of three esthetic resin composite materials. Oper Dent. 2004;29(2):203-11.

9. Baseren M. Surface roughness of nanofill and nanohybrid composite resin and ormocer-based tooth-colored restorative materials after several finishing and polishing procedures. J Biomater Appl. 2004;19(2):121-34.

10.Irie M, Tjandrawinata R, Suzuki K. Effect of delayed polishing periods on interfacial gap formation of class V restorations. Oper Dent. 2003; 28(5):552-9.

11. Roeder LB, Tate WH, Powers JM. Effect of finishing and polishing procedures on the surface roughness of packable composites. Oper Dent. 2000; 25(6):534-43.

12.Ryge G, Foley DE, Fairhurst CW. Micro-indentation hardness. J Dent Res. 1961; 40(6):1116-26.

13.Irie M, Suzuki K. Effects of delayed polishing on gap formation of cervical restorations. Oper Dent. 2002; 27(1):59-65.

14. Stanford WB, Fan PL, Wozniak WT, Stanford JW. Effects of finishing on color and gloss of composites with different fillers. J Am Dent Assoc. 1985; 110(2):211-3.
15.Anusavice KJ. Physical Properties of Dental Materials in Philip's science of dental materials. 10th ed. Philadephia: Saunders, 2003. p. 43.

16.Craig RG, Ward ML. Restorative dental materials. 10th ed. St. Louis, USA: Mosby; 1997.

17. Scheibe KG, Almeida KG, Medeiros IS, Costa JF, Alves CM. Effect of different polishing systems on the surface roughness of microhybrid composites. J Appl Oral Sci. 2009;17(1):21-6.

18.Park S, Krejci I, Lutz F. Hardness of celluloid stripfinished or polished composite surfaces with time. J Prosthet Dent. 2000;83(6):660-3.

19. Von Fraunhofer JA, Curtis P Jr. The physical and mechanical properties of anterior and posterior composite restorative materials. Dent Mater. 1989;5(6):365-8.

20.Chinelatti MA, Chimello TD, Ramos PR. Avaliação da dureza superficial de resinas compostas antes e após o polimento em diferentes tempos. J Appl Oral Sci. 2006;14(3):188-92.

21. Yazici A, Tuncer D, Onen A, Kilinc E. Effects of delayed finishing/polishing on surface roughness, hardness and gloss of tooth-coloured restorative materials. Eur J Dent. 2010;4(1):50-6.

22. Yap AUJ, Sau CW, Lye KW. Effects of finishing/ polishing time on surface characteristics of toothcoloured restoratives. J Oral Rehabil. 1998;25(6):45661.

23. Venturini D, Cenci M, Demarco F, Camacho G, Powers J. Effect of polishing techniques and time on surface roughness, hardness and microleakage of resin composite restorations. Oper Dent. 2006;31(1):11-7.

24. Venturini D, Cenci M, Demarco F, Camacho G, Powers J. The effect of polishing techniques and time on the surface characteristics and sealing ability of resin composite restorations after one-year storage. Oper Dent. 2008;33(2):169-76

Recibido : 12/08/2013

Aceptado: 18/10/2013 